\section{Cancer stress tests}

\section{By Joanne Kotz, Senior Editor}

Two research teams have separately described distinct ways to block the unfolded protein response, a prosurvival pathway that is often upregulated in cancers in response to endoplasmic reticulum stress. Both efforts center on inhibiting an endoplasmic reticulum transmembrane protein called IRE1. MannKind Corp. is blocking IRE1's ribonuclease domain, ${ }^{1}$ while U.K. academics are targeting the protein's kinase domain. ${ }^{2}$

MannKind plans to move its lead IRE1 (endoplasmic reticulum to nucleus signaling 1; ERN1) ribonuclease (RNase) inhibitor into the clinic in multiple myeloma (MM) in 2012. The U.K. team is identifying drug leads against the kinase domain.

Hypoxic conditions, nutrient deprivation or protein folding disturbances can lead to the accumulation of unfolded proteins in the endoplasmic reticulum (ER). In tumors, for example, rapid proliferation and poor vascularization can lead to substantial ER stress.

To prevent this ER stress from leading to cell death, cells trigger an unfolded protein response (UPR) that is orchestrated by a transcription factor called $\mathrm{x}$-box binding protein 1 (XBP1).

IRE1 plays a pivotal role in kick-starting the UPR. ER stress triggers the dimerization of IRE1, which in turn causes the proteins' kinase domains to autophosphorylate. Phosphorylation activates the IRE1 RNase domain, which then splices the mRNA of XBP1 into its final, active form (see Figure 1, "Blocking the unfolded protein response in cancer").

MM is particularly dependent on activation of the UPR. XBP1 is frequently overexpressed in MM patients, and overexpression of Xbp1 in mice leads to an MM-like disease. ${ }^{3}$

"Clearly, the results of numerous researchers have confirmed that $\mathrm{XBP} 1$ plays a significant role in the pathology of multiple myeloma, an incurable plasma cell malignancy," said John Patterson, associate director at MannKind and project leader of oncology lead discovery.

Because IRE1 splicing directly regulates XBP1 levels, MannKind embarked on a screening program to identify inhibitors of the IRE1 RNase domain. In a high throughput assay, the company discovered a series of salicylaldehyde analogs that inhibited IRE1 cleavage of an XBP1 mRNA substrate. In human MM cells and in mice under ER stress, the most potent compound inhibited XBP1 mRNA splicing compared with vehicle controls.

Data were published in The Journal of Biological Chemistry.

The company has optimized those hits and now has a series of lead compounds that are "highly potent and selective for the target in vitro and in vivo, that are orally dosed and that have excellent pharmacokinetics and ADMET characteristics," said Patterson.

MannKind hopes to start clinical trials of its most advanced IRE1

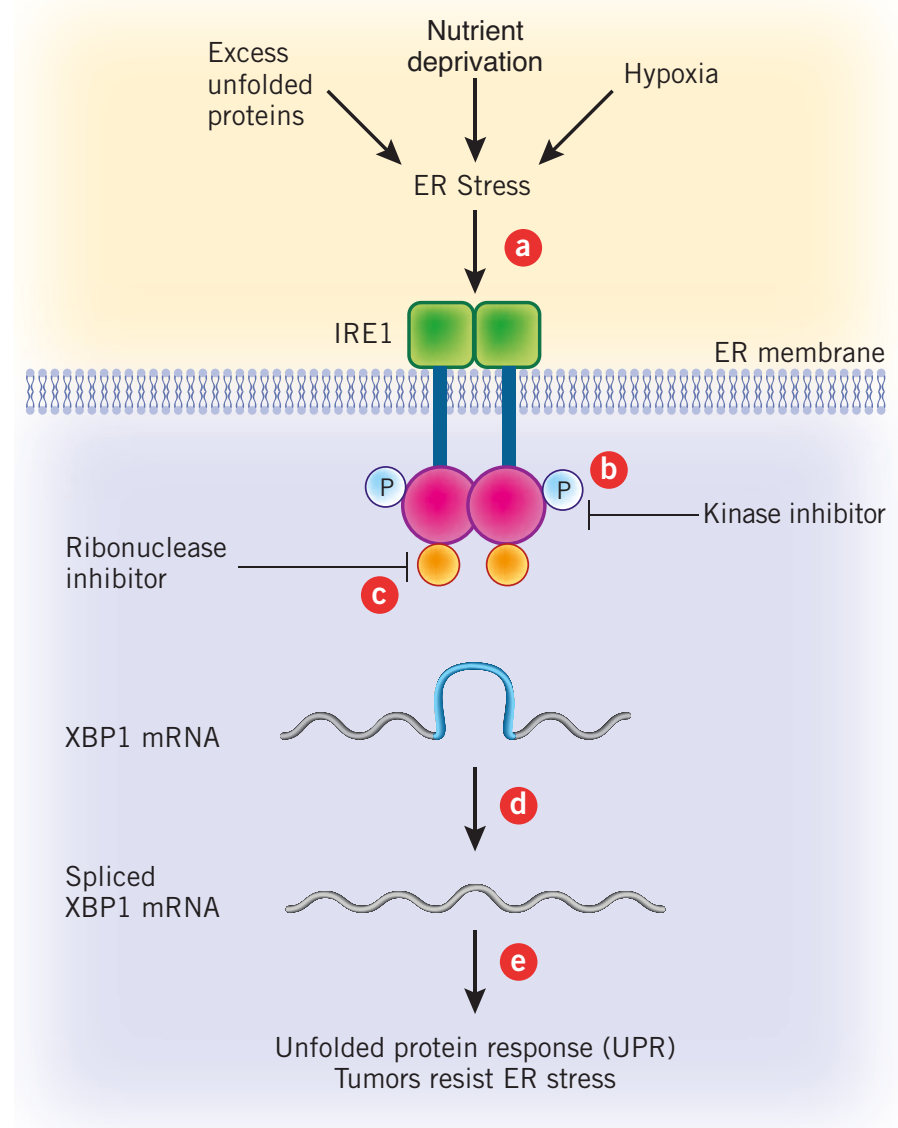

Figure 1. Blocking the unfolded protein response in cancer. In tumors, excess levels of unfolded proteins, nutrient deprivation or hypoxia can trigger endoplasmic reticulum (ER) stress. This ER stress is sensed by endoplasmic reticulum to nucleus signaling 1 (ERN1; IRE1) through the ER-localized domain (green) and leads to dimerization of the transmembrane protein [a]. IRE1 dimerization causes the cytosolic kinase domain (pink) to autophosphorylate $(\mathrm{P})[\mathrm{b}]$, leading to activation of the ribonuclease (RNase) domain (orange) [c]. The active IRE1 RNase domain removes an intron from the mRNA of $\mathrm{x}$-box binding protein 1 (XBP1) [d]. This spliced mRNA is translated into the XBP1 transcription factor, which induces expression of genes involved in the unfolded protein response (UPR) [e]. In some cancers, the IRE1-XBP1 pathway is upregulated to promote survival. In The Journal of Biological Chemistry, researchers at MannKind Corp. described IRE1 RNase inhibitors that block XBP1 mRNA splicing. In The EMBO Journal, a U.K. team reported that inhibiting the IRE1 kinase domain also prevents IRE1-mediated splicing.

RNase inhibitor, MKC204, in MM in 2012. Its preclinical work is studying the inhibitors in additional oncology indications.

"Any tumor that is under stress requires robust adaptive responses to survive. Some of these adaptive responses are mediated through the ER, so the selective targeting of the ER response will likely be effective 
in a wide variety of tumor types," said Ray Tabibiazar, president and CEO of Ruga Corp.

A 2010 Blood paper by Ruga cofounder Albert Koong, an associate professor of radiation oncology at Stanford University, showed that an IRE1 RNase inhibitor decreased tumor size compared with a vehicle control in a mouse xenograft model of human MM. ${ }^{4}$

Ruga has licensed an IRE1 RNase inhibitor and an XBP1 transgenic mouse model from Stanford for a preclinical program targeting the IRE1 RNase domain and is aiming for an IND filing in 2013.

"The Blood paper clearly showed that the IRE1 ribonuclease domain is a druggable target and the inhibitor has in vivo activity at well-tolerated doses. The XBP1 transgenic mouse in that paper has proven to be an invaluable tool for in vivo assessment of such drugs as well as other drugs that affect the ER stress pathways," said Tabibiazar.

"These two papers show that selective targeting of IRE1 ribonuclease activity is possible," agreed David Ron, professor of cellular pathophysiology and clinical biochemistry at the University of Cambridge. "It would be nice if we understood how such inhibition is attained, for instance the site of action of the inhibitors, but the papers provide almost no clues."

Thus, Ron wanted to see a more detailed characterization of the mechanism of RNase inhibition.

\section{Kinase in the mix}

Although blocking a target's kinase domain is usually the preferred path for drug developers, IRE1 kinase inhibitors have been off the table since 2003, when reports showed that inhibiting the yeast Irel's kinase domain actually activated the RNase domain. ${ }^{5,6}$

"Researchers were frightened off targeting the kinase domain by the yeast work," said Laurence Pearl, professor of structural biology in the Genome Damage and Stability Centre and head of the School of Life Sciences at the University of Sussex.

Now, a team led by Pearl, Faith Davies and Maruf Ali has shown that with human IRE1, the exact opposite occurs-inhibiting the kinase domain leads to inhibition of the RNase domain.

Davies is a consultant hematologist leading the myeloma targeted treatment team at The Institute of Cancer Research, and Ali has a Cancer Research UK research fellow in the Division of Molecular Biosciences at the Imperial College London.

In human myeloma cells under ER stress, an inhibitor of IRE1 kinase prevented XBP1 mRNA splicing and decreased XBP1 protein levels compared with no treatment.

The work, which is not patented, was reported in The EMBO Journal.

"Our results suggest that an IRE1 kinase inhibitor and RNase inhibitor are likely to have similar therapeutic effect," said Pearl. "The advantage of a kinase as a target is that we know so much about developing kinase inhibitors. With the IRE1 ribonuclease you have significantly more challenges, including not currently knowing the enzyme's mechanism and not even knowing the exact location and functional state of the RNase active site."

The U.K. team is developing selective IRE1 kinase inhibitors. Pearl said the group has identified hits and now plans to optimize these compounds and test them in preclinical models of MM.

\section{Feeling the stress}

Beyond cancer, ER stress occurs in a number of pathologies that involve high levels of secreted proteins or the accumulation of misfolded proteins. ${ }^{7}$
"Other conditions where ER stress is associated with disease, and which might therefore be opportunities, include Parkinson's disease, Alzheimer's disease, polyglutamine diseases, diabetes, diabetic retinopathy, dyslipidemia and inflammatory diseases," said Walter Newman, head of external innovation at Proteostasis Therapeutics Inc.

Peter Reinhart, president and CSO at Proteostasis, told SciBX that the therapeutic rationale for targeting the UPR to decrease the accumulation of aggregated proteins or toxic intermediates in neurodegenerative diseases is particularly strong. "The UPR is a complex response, and tweaking the system a little bit may produce significant effects. We are beginning to understand how some of our protein proteostasis regulator molecules might achieve therapeutically meaningful results for specific diseases," he said.

In November 2010, Proteostasis initiated a collaboration with Laurie Glimcher to look at the role of XBP1 in neurodegenerative diseases, said Reinhart. Glimcher is professor of immunology at the Harvard School of Public Health and professor of medicine at Harvard Medical School.

Proteostasis has an ongoing program aimed at discovering small molecule modulators of the UPR for the treatment of neurodegenerative and orphan diseases.

MannKind is also investigating its IRE1 RNase inhibitors in indications outside cancer, including neurodegenerative diseases, metabolic diseases, viral infections and inflammation.

MannKind holds a composition-of-matter patent on its IRE1 RNase inhibitors, and Patterson said the company is in discussions with potential partners.

Kotz, J. SciBX 4(10); doi:10.1038/scibx.2011.270 Published online March 10, 2011

\section{REFERENCES}

1. Volkmann, K. et al. J. Biol. Chem.; published online Feb. 8, 2011; doi:10.1074/jbc.M110.199737

Contact: John B. Patterson, MannKind Corp., Valencia, Calif. e-mail: jpatterson@mannkindcorp.com

2. Ali, M.M.U. et al. EMBO J.; published online Feb. 11, 2011; doi:10.1038/emboj.2011.18

Contact: Laurence H. Pearl, University of Sussex, Brighton, U.K. e-mail: laurence.pearl@sussex.ac.uk

Contact: Faith E. Davies, The Institute of Cancer Research,

Surrey, U.K

e-mail: faith.davies@icr.ac.uk

Contact: Maruf M.U. Ali, Imperial College London, London, U.K. e-mail: maruf.ali@imperial.ac.uk

3. Carrasco, D.R. et al. Cancer Cell 11, 349-360 (2007)

4. Papandreou, I. et al. Blood 117, 1311-1314 (2011)

5. Papa, F.R. et al. Science 302, 1533-1537 (2003)

6. Korennykh, A.V. et al. Nature 457, 687-693 (2009)

7. Hetz, C. \& Glimcher L.H. Mol. Cell 35, 551-561 (2009)

\section{COMPANIES AND INSTITUTIONS MENTIONED \\ Cancer Research UK, London, U.K. \\ Harvard Medical School, Boston, Mass. \\ Harvard School of Public Health, Boston, Mass. \\ Imperial College London, London, U.K. \\ The Institute of Cancer Research, Surrey, U.K. \\ MannKind Corp. (NASDAQ:MNKD), Valencia, Calif. \\ Proteostasis Therapeutics Inc., Cambridge, Mass. \\ Ruga Corp., Palo Alto, Calif. \\ Stanford University, Stanford, Calif. \\ University of Cambridge, Cambridge, U.K. \\ University of Sussex, Brighton, U.K.}

